UCSBTH-98-01

hep-th/9801074

\title{
Black Holes with Multiple Charges and the Correspondence Principle
}

\author{
HAISONG YANG1 \\ Department of Physics, University of California, Santa Barbara, CA 93106, USA
}

\begin{abstract}
We consider the entropy of near extremal black holes with multiple charges in the context of the recently proposed correspondence principle of Horowitz and Polchinski, including black holes with two, three and four Ramond-Ramond charges. We find that at the matching point the black hole entropy can be accounted for by massless open strings ending on the D-branes for all cases except a black hole with four Ramond-Ramond charges, in which case a possible resolution in terms of brane-antibrane excitations is considered.
\end{abstract}

January, 1998

1 E-mail: yangh@cosmic1.physics.ucsb.edu 


\section{Introduction}

A few years ago, Susskind proposed [1] that there should be a one-to-one correspondence between quantum states of a Schwarzschild black hole and fundamental string states. This idea was carried out further in [2] [3]. However, it was believed that there was a discrepancy between these two objects: the black hole entropy scales as $M^{2}$ while the entropy of a fundamental string scales as $M$. Recently, Horowitz and Polchinski formulated a correspondence principle [4] for black holes and strings which resolves the discrepancy in a certain way. It states that quantum states of black holes can be matched onto states of strings and D-branes [5] at a special point of the string coupling constant $g$ and during the transition the mass changes by at most a factor of order unity.

More precisely, the correspondence principle works in the following way. Start with the black hole picture. Decrease $g$ until the curvature (in the string metric) at the event horizon becomes of order the string scale. At this point (called the matching point), the $\alpha^{\prime}$ corrections in the low energy effective action become important and the black hole solution can not be trusted any more. So, the black hole is gone and the physical picture changes, what takes the place of the black hole is some description in terms of strings and D-branes, since these are the dynamic degrees of freedom that we have. On the black hole side there is only a thermodynamic description, while on the string side there is also a statistical description, which enables one to calculate the entropy microscopically. What was found is that the thermodynamic quantities, such as entropy, temperature, etc., of these two pictures agree with each other. Of course, we do not have a precise description of the transition regime, so the matching is only up to factors of order unity.

In [4], three different cases were considered: Schwarzschild black hole, near extremal black holes with fundamental string winding and momentum charge, near extremal black p-brane with a single RR(Ramond-Ramond) charge. Agreement was found for all three cases. Notice that for the last two cases, if the black holes are not near extremal, i.e., if the charges that they carry are small, then they are qualitatively the same as a Schwarzschild black hole, since the correspondence principle is not sensitive to factors of order unity.

At the matching point, the Schwarzschild black hole is described by a long selfinteracting fundamental string that has size of order $\alpha^{1 / 2}$. The second black hole is described by an excited fundamental string that is wrapped around a $S^{1}$ and also carries some momentum in the same direction. The black p-branes are described by D p-branes wrapped around the internal torus with some excitations described by massless open strings 
ending on the D-branes. It was thought in [4] that there is a large gravitational field dressing for all $p$-branes at the matching point. We will show that while this is true for $p=4,5,6$, there is no large dressing for $p=0,1,2,3$.

It is natural to ask whether the correspondence principle can be applied to other black holes. An important class of black holes are the ones that carry multiple RR charges in type IIA or type IIB string theory. If the correspondence principle can be applied to them, then at the matching point, they should all be described by certain D-brane configurations with some excitations.

D-branes have even spatial dimensions in type IIA and odd spatial dimensions in type IIB. Upon compactification on a torus, T-duality interchanges type IIA and type IIB, even dimensional D-branes and odd dimensional D-branes. If we have two kinds of D-branes, the number of ND (Neumann-Dirichlet) type of boundary conditions between them, however, is invariant under T-duality. So black holes that carry two RR charges can be put into the following three categories according to the the number of ND conditions:

(A) 2 ND conditions

(B) 4 ND conditions

(C) 6 ND conditions.

A black hole with D 0- and D 6-brane charges was examined in [6] in the context of the correspondence principle and agreement was found. In this paper, we consider all black holes in category (A) and (B). We also consider black holes with three or four RR charges.

The plan of this paper is the following. In section II we examine the issue of gravitational field dressing of the black p-branes with a single RR charge. In section III we first consider a five dimensional black hole with D 1- and D 5-brane charges and a six dimensional black hole with D 0- and D 4-brane charges, both of which belongs to category (B). We also include the discussion of a four dimensional black hole that carries D 2-brane, D 6-brane and NS(Neveu-Schwarz) 5-brane charges because it shares some common feature with the above two black holes. In section IV, we consider a four dimensional black hole that can carry up to four RR charges. It is a black hole in category (B) if two of the charges are set to be zero. We also consider the case when three or four charges are large. In section V, we consider an eight dimensional black hole that carries D 0- and D 2-brane charges, which belongs to category (A). We also discuss a generalization of this black hole. We conclude in section VI. 
Other aspects of the correspondence principle were examined in [7].

\section{Black Holes with a Single RR Charge}

In this section, we consider the black $p$-branes with a single $\mathrm{RR}$ charge. In [4] it was noticed that if one starts with D-branes in flat spacetime at zero string coupling and increases the coupling constant, then gravitational effects become important before the black hole is formed. In particular the locally measured size of the internal torus and temperature can differ from their asymptotic values by a large factor. We show that while this large gravitational field dressing is indeed present for $p=4,5,6$, there is no large gravitational dressing for $p=0,1,2,3$. The conclusion that the entropy can be accounted for by the massless open strings however is still correct, as we will see.

The string metric of the black p-branes is given by

$$
d s^{2}=H^{-1 / 2}\left[-\left(1-\frac{r_{0}^{n}}{r^{n}}\right) d t^{2}+d y^{a} d y_{a}\right]+H^{1 / 2}\left[\left(1-\frac{r_{0}^{n}}{r^{n}}\right)^{-1} d r^{2}+r^{2} d \Omega_{n+1}^{2}\right]
$$

where

$$
H=1+\frac{r_{0}^{n} \sinh ^{2} \alpha}{r^{n}}
$$

The $y_{a}$ are $p=7-n$ spatial coordinates along the brane which we assume are compactified on a torus. The dilaton is $e^{2 \phi}=H^{(n-4) / 2}$. The horizon is at $r=r_{0}$.

Let us define $r^{*}=r_{0}(\cosh \alpha)^{2 / n}$, the point where $H$ becomes of order 1 . The crucial thing to notice is that the spatial geometries outside the horizon are different for different $p$-branes. The angular part of metric is $\left(1+\frac{r_{0}^{n} \sinh ^{2} \alpha}{r^{n}}\right)^{1 / 2} r^{2} d \Omega_{n+1}^{2}$. If $n<4(p=4,5,6)$, the radius of the $S_{n+1}$ increases with some power in $r$ outside the horizon. If $n=4(p=3)$, the radius of the $S_{5}$ does not change much for $r_{0}<r<r^{*}$ and increases linearly with $r$ for $r>r^{*}$. In other words, there is a deep throat from $r_{0}$ to $r^{*}$. If $n>4(p=0,1,2)$, the radius of the $S_{n+1}$ first decreases, it reaches the minimum at $r \sim r^{*}$ and then increase linearly with $r$. We will refer to this kind of geometry as the "bottle neck".

By the correspondence principle the matching between black holes and D-branes and strings happens when the curvature at the horizon becomes of order the string scale. For these black p-branes, the largest contribution to the curvature comes from the angular part of the metric. This means the matching happens when the radius of the $S_{n+1}$ is of order $\alpha^{1 / 2}$. We immediately see the difference between different $p$-branes. For $p=4,5,6$, 
the stringy behavior only smears out to $r \sim r_{0}$ ( the zero in $\left(1-\frac{r_{0}^{n}}{r^{n}}\right)$ is smeared out). So a large gravitational field dressing is left and the local temperature is of order the string scale due to the redshift. For $p=3$, the stringy behavior not only smears out the horizon, but also the whole throat and the metric (2.1) can only be trusted for $r>r^{*}$. As a result, there is no large dressing for the 3-brane and the spacetime is basically flat. Similarly, for $p=0,1,2$, the stringy behavior smears out the metric all the way to $r \sim r_{0}(\cosh \alpha)^{\frac{3}{n+2}}$, where the curvature becomes of order the string scale again. We can imagine the process of approaching the matching point from the black hole side for the last case. The fundamental string length grows relative to the size of the black hole as we decrease the coupling constant. The stringy behavior should first smears out the region at the minimum radius $r \sim r^{*}$, the geometry there gets corrected but the black hole still exists. As the coupling constant is further decreased, the smeared out region will grow and eventually, at the matching point, the horizon is smeared out. Since there is no large dressing for $p \leq 3$, the local temperature is the same as asymptotic temperature and is small in string units.

The above observation nevertheless does not jeopardize the conclusion that the entropy of all $p$-branes can be accounted for by the massless open strings on the D-branes. A priori, we should do the calculation locally at the D-branes. However, it was pointed out in [4] that in fact one can do the calculation either locally or at $r=\infty$, which follows from the observation that the entropy and energy expressions of the ideal gas remain the same under the rescaling due the large dressing. We have just seen that there is no large dressing for black p-branes with $p \leq 3$, so we should use the asymptotic quantities to do the calculation for them anyway. The conclusion therefore is unaffected. We should remark that this is the special property of black holes with a single RR charge. For black holes with more than one charge, in general we can only do the calculation locally.

\section{Three Black Holes with Two or Three Charges}

In this section, we consider three different black holes: a five dimensional black hole with D 1- and D 5-brane charges, a six dimensional black hole with D 0- and D 4-brane charges and a four dimensional black hole with D 2-brane, D 6-brane and NS 5-brane charges. All charges are assumed to be large. Although the precise counting of entropy has already been done for the first and third black hole (but not for the second one), we want to apply the correspondence principle to all of them as a test for the correspondence 
principle. We will see that it can be applied to all three black holes successfully. In particular, we resolve a puzzle discussed in [4].

We consider these black holes together in this section because they share some common features. For all three black holes, the spatial geometry outside the horizon is either a deep throat or a "bottle neck", which are smeared out by the stringy behavior at the matching point. This reduces the redshift effect and causes the local temperatures to be small in string scale. These features are similar to the black p-branes with $p \leq 3$ discussed in the last section. On the other hand, these black holes carry more than one charge, so there could still be large gravitational field dressing, which depends on the relative magnitudes of the boost parameters. This will become clear in later discussions.

In the following, we first consider the five dimensional black hole with D 1- and D 5 -brane charges in detail in 3.1. We then briefly discuss the other two black holes in 3.2 and 3.3, in particular the new features of each one.

\subsection{Five Dimensional Black Hole with D 1-and D 5-brane Charges}

The string metric of the five dimensional black hole with D 1- and D 5-brane charges is given by

$$
\begin{aligned}
d s^{2}= & \left(H_{1} H_{5}\right)^{1 / 2}\left[-\left(H_{1} H_{5}\right)^{-1} f d t^{2}+\left(H_{1} H_{5}\right)^{-1} d y_{1}^{2}+H_{5}^{-1}\left(d y_{2}^{2}+d y_{3}^{2}+d y_{4}^{2}+d y_{5}^{2}\right)\right. \\
& \left.+f^{-1} d r^{2}+r^{2} d \Omega_{3}^{2}\right]
\end{aligned}
$$

where

$$
\begin{aligned}
H_{i} & =1+\frac{r_{0}^{2} \sinh ^{2} \alpha_{i}}{r^{2}} \quad(i=1,5) \\
f & =1-\frac{r_{0}^{2}}{r^{2}}
\end{aligned}
$$

The $y_{a}$ are coordinates on the internal $T^{5}$ which are identified with length $L_{a}$. The dilaton is $e^{2 \phi}=H_{1} H_{5}^{-1}$

This black hole is a solution to the type IIB string theory on $T^{5}$ and can be constructed by the 'harmonic function' rule described in [8]. The horizon is at $r=r_{0}$. The extremal limit of the black hole can be obtained by letting $r_{0} \rightarrow 0$ while fixing $r_{0}^{2} \sinh ^{2} \alpha_{i}$ and has zero horizon area. The extremal limit preserves $1 / 4$ of the supersymmetries and can be thought of as a superposition of extremal black 5-branes wrapped around the $T^{5}$ and black 1-branes wrapped along the $y_{1}$ direction and smeared out in the rest four internal directions. The black hole is near extremal when at least one RR charge is large (i.e., one 
$\cosh \alpha_{i}$ is much larger than 1). Near extremal black holes can be thought of as excited states of the extremal limit. If only one charge is large, the black hole is essentially the same as the black holes with a single charge, since the correspondence principle would not be able to distinguish them. We will consider the case when both charges are large.

The energy, excess energy, entropy, Hawking temperature and two RR charges of the black hole are

$$
\begin{aligned}
E & \sim \frac{L_{1} L_{2} L_{3} L_{4} L_{5}}{g^{2} \alpha^{\prime 4}} r_{0}^{2}\left(\cosh 2 \alpha_{1}+\cosh 2 \alpha_{5}+1\right) \\
\Delta E_{b h} & \sim \frac{L_{1} L_{2} L_{3} L_{4} L_{5}}{g^{2} \alpha^{\prime 4}} r_{0}^{2} \\
S_{b h} & \sim \frac{L_{1} L_{2} L_{3} L_{4} L_{5}}{g^{2} \alpha^{\prime 4}} r_{0}^{3} \cosh \alpha_{1} \cosh \alpha_{5} \\
T & \sim \frac{1}{r_{0} \cosh \alpha_{1} \cosh \alpha_{5}} \\
Q_{1} & \sim \frac{L_{2} L_{3} L_{4} L_{5}}{g \alpha^{\prime 3}} r_{0}^{2} \sinh 2 \alpha_{1} \\
Q_{5} & \sim \frac{1}{g \alpha^{\prime}} r_{0}^{2} \sinh 2 \alpha_{5}
\end{aligned}
$$

The excess energy $\Delta E_{b h}$ is the energy above the BPS limit. We have dropped the overall numerical coefficients in these expressions since the correspondence principle is not sensitive to them. However, the coefficients in front of $E$ and $Q_{i}$ are the same, so that $\Delta E_{b h}$ takes the above form. For near BPS black holes, the correspondence principle states that the excess energy changes by at most a factor of unity during the transition. This will be used when we calculate the entropy on the string side.

The matching between the black hole states and states of D-branes and strings occurs when the curvature at the horizon is of order $1 / \alpha^{\prime}$. The largest contribution comes from the angular part of the metric and is of order $\left(r_{0}^{2} \cosh \alpha_{1} \cosh \alpha_{5}\right)^{-1}$. So the matching point is

$$
r_{0} \sim \frac{\alpha^{\prime 1 / 2}}{\left(\cosh \alpha_{1} \cosh \alpha_{5}\right)^{1 / 2}}
$$

Let us define $r^{*}=r_{0} \cosh \alpha_{\text {min }}$, where $\cosh \alpha_{\text {min }}$ is the smaller one of $\cosh \alpha_{1}$ and $\cosh \alpha_{5}$. The spatial geometry outside the horizon has a deep throat from $r_{0}$ to $r^{*}$, which is smeared out by stingy behavior at the matching point. If $\cosh \alpha_{1} \sim \cosh \alpha_{5}$, we have $H_{1}\left(r=r^{*}\right) \sim H_{5}\left(r=r^{*}\right) \sim 1$ and there is no large gravitational field dressing; if there is a hierarchy between $\cosh \alpha_{1}$ and $\cosh \alpha_{5}$, then there is still a large dressing. In either case, we can write $H_{i}\left(r=r^{*}\right) \sim \frac{\cosh ^{2} \alpha_{i}}{\cosh ^{2} \alpha_{m i n}},(i=1,5)$. In the following, we will treat both cases 
together, all formulas will be be valid for both. It is instructive to write down the metric at the D-branes (i.e., $\left.r=r^{*}\right)$ :

$$
\begin{gathered}
d s^{2} \sim\left(\frac{\cosh \alpha_{1} \cosh \alpha_{5}}{\cosh ^{2} \alpha_{\min }}\right)\left[-\frac{d t^{2}}{\left(\frac{\cosh \alpha_{1} \cosh \alpha_{5}}{\cosh ^{2} \alpha_{m i n}}\right)^{2}}+\frac{d y_{1}^{2}}{\left(\frac{\cosh \alpha_{1} \cosh \alpha_{5}}{\cosh ^{2} \alpha_{m i n}}\right)^{2}}\right. \\
\left.+\frac{d y_{2}^{2}+d y_{3}^{2}+d y_{4}^{2}+d y_{5}^{2}}{\left(\frac{\cosh \alpha_{5}}{\cosh \alpha_{m i n}}\right)^{2}}+\left(\frac{\cosh ^{2} \alpha_{m i n}}{\cosh \alpha_{1} \cosh \alpha_{5}}\right) \alpha^{\prime} d \Omega_{3}^{2}\right]
\end{gathered}
$$

where we have substituted (3.4) into the metric. Let us use $T^{\prime}, \Delta E_{b h}^{\prime}, L_{a}^{\prime}$ to denote the local temperature, excess energy and spatial sizes of the $T^{5}$. Following (3.3) (3.4) (3.5), they are given by

$$
\begin{aligned}
T^{\prime} & \sim \frac{1}{\alpha^{\prime 1 / 2} \cosh \alpha_{\min }} \\
\Delta E_{b h}^{\prime} & \sim \frac{L_{1} L_{2} L_{3} L_{4} L_{5}}{g^{2} \alpha^{\prime 3}\left(\cosh \alpha_{1} \cosh \alpha_{5}\right)^{1 / 2} \cosh \alpha_{m i n}} \\
L_{1}^{\prime} & \sim L_{1} \frac{\cosh \alpha_{m i n}}{\left(\cosh \alpha_{1} \cosh \alpha_{5}\right)^{1 / 2}} \\
L_{i}^{\prime} & \sim L_{i}\left(\frac{\cosh \alpha_{1}}{\cosh \alpha_{5}}\right)^{1 / 2}(i=2,3,4,5)
\end{aligned}
$$

These expressions are correct independent of whether there is large dressing or not. At the matching point, the entropy and charges are

$$
\begin{aligned}
S_{b h} & \sim \frac{L_{1} L_{2} L_{3} L_{4} L_{5}}{g^{2} \alpha^{\prime 5 / 2}\left(\cosh \alpha_{1} \cosh \alpha_{5}\right)^{1 / 2}} \\
Q_{1} & \sim \frac{L_{2} L_{3} L_{4} L_{5} \cosh \alpha_{1}}{g \alpha^{\prime 2} \cosh \alpha_{5}} \\
Q_{5} & \sim \frac{\cosh \alpha_{5}}{g \cosh \alpha_{1}}
\end{aligned}
$$

Now let us consider the statistical description of the system. It should be described by $Q_{1} \mathrm{D}$ 1-branes and $Q_{5} \mathrm{D}$ 5-branes with some excitations. We will assume the excitations are described by massless open strings on the D-branes and see that it gives consistent result. As we discussed at the end of section 2 , the calculation has to be done locally at the D-branes.

There are three kinds of open strings : 1-1, 5-5 and 1-5 strings. For fixed amount of excess energy $\Delta E_{b h}^{\prime}$, one can ask the following question: to maximize the entropy, what is the correct distribution of $\Delta E_{b h}^{\prime}$ among the three kinds of open strings. The answer is that 
the three kinds of open strings should have the same temperature. It turns out that if we put $\Delta E_{b h}^{\prime}$ into the open strings, we get a local temperature that is the same as the $T^{\prime}$ in (3.6). This is part of the matching property that we would like to have. To facilitate the calculation, we will just assume the local temperature to be $T^{\prime}$ and calculate the entropy and energy carried by these open strings. We will see that they agree with $S_{b h}$ and $\Delta E_{b h}^{\prime}$.

Let us use $S_{i j}$ to denote the entropy contribution from the $i-j$ strings. We consider the 5-5 strings first. The 5-5 strings are a five dimensional gas at local temperature $T^{\prime}$. The number of species of open strings is $Q_{5}^{2}$. So the entropy is

$$
S_{55} \sim Q_{5}^{2} L_{1}^{\prime} L_{2}^{\prime} L_{3}^{\prime} L_{4}^{\prime} L_{5}^{\prime} T^{\prime 5} \sim \frac{S_{b h}}{\cosh ^{4} \alpha_{\min }}
$$

The second step follows from direct substitutions of (3.6) and (3.7). The above picture is not valid if $T^{\prime} L_{i}^{\prime}<1$ for any $L_{i}^{\prime}$, since the open strings do not see those dimensions and behave like a lower dimensional gas. In such case, we should use the wrapped picture to calculate the entropy, i.e., let the D-branes be wrapped around those small circles many times(See [4] for detailed discussions). This will increase the effective volume of the gas and reduce the number of species of strings. The net result is that equation (3.8) is still valid and gives the correct entropy.

Next, we consider 1-1 strings. We can avoid the calculation if we notice the T-dual relationship between the 1-1 and 5-5 strings. If we T-dualize the $y_{2}, y_{3}, y_{4}, y_{5}$ directions, the 5 -branes become 1-branes and 5-5 strings become 1-1 strings and vice versa. But the $S_{55}$ expression $\frac{S_{b h}}{\cosh ^{4} \alpha_{m i n}}$ is invariant under this operation, so we must have $S_{11} \sim S_{55}$.

Although not necessary, it is still instructive to calculate $S_{11}$ directly in the original picture. We need to first determine the number of species of 1-1 strings that are excited. The $Q_{1} \mathrm{D}$ 1-branes are evenly distributed in the $y_{2}, y_{3}, y_{4}, y_{5}$ directions. Let us focus on the $y_{2}$ direction for now. Because the local temperature is of order $T^{\prime}$, the open strings that are excited should have length of order $\alpha^{\prime} T^{\prime}$ or smaller. An open string that starts from a particular 1-brane can end on any 1-brane within $\alpha^{\prime} T^{\prime}$ distance, so the number of 1-branes that it can end on is $Q_{1} \frac{\alpha^{\prime} T^{\prime}}{L_{2}^{\prime}}$. The total number of 1-1 strings that are excited is therefore $Q_{1}^{2} \frac{\alpha^{\prime} T^{\prime}}{L_{2}^{\prime}}$. We can also try to understand the factor $\frac{\alpha^{\prime} T^{\prime}}{L_{2}^{\prime}}$ in the T-dual picture. If we T-dualize the $y_{2}$ direction, the 1-branes becomes 2-branes. We have a two dimensional open string gas at the same temperature $T^{\prime}$. The number of species is now simply $Q_{1}^{2}$, but the spatial size of the 2-branes in the $y_{2}$ direction becomes $\frac{\alpha^{\prime}}{L_{2}^{\prime}}$, which when combined with the extra $T^{\prime}$ gives us the same factor. The same consideration applies to $y_{3}, y_{4}, y_{5}$ 
directions. So, there are $Q_{1}^{2} \frac{\alpha^{\prime 4} T^{\prime 4}}{L_{2}^{\prime} L_{3}^{\prime} L_{4}^{\prime} L_{5}^{\prime}}$ species of open strings. Of course, from the 1-brane world volume point of view, the masses of these open strings range from zero to $T^{\prime}$. But to the accuracy of factors of order unity, we can treat them as massless. So the entropy from 1-1 strings is

$$
S_{11} \sim Q_{1}^{2} \frac{\alpha^{\prime 4} T^{\prime 4}}{L_{2}^{\prime} L_{3}^{\prime} L_{4}^{\prime} L_{5}^{\prime}} T^{\prime} L_{1}^{\prime} \sim \frac{S_{b h}}{\cosh ^{4} \alpha_{\min }}
$$

Now let us consider the 1-5 strings. The 1-5 open strings have NN(NeumannNeumann) boundary condition in the $y_{1}$ direction and ND or DN boundary condition in the $y_{2}, y_{3}, y_{4}, y_{5}$ directions. It is a one dimensional gas. The ND or DN boundary condition does not change the number of species of the strings, so there are $Q_{1} Q_{5}$ of them. The entropy from the 1-5 strings is then

$$
S_{15} \sim Q_{1} Q_{5} T^{\prime} L_{1}^{\prime} \sim S_{b h}
$$

The total entropy $S_{\text {open }}$ and total local energy $E_{\text {open }}^{\prime}$ carried the three kinds open strings are

$$
\begin{aligned}
& S_{\text {open }}=S_{11}+S_{55}+S_{15} \sim S_{15} \sim S_{b h} \\
& E_{\text {open }}^{\prime} \sim T^{\prime} S_{\text {open }} \sim \Delta E_{b h}^{\prime}
\end{aligned}
$$

The relation between $E_{\text {open }}^{\prime}$ and $S_{\text {open }}$ just follows from the fact that we have an open string gas at local temperature $T^{\prime}$. The fact that $E_{\text {open }}^{\prime}$ agrees with $\Delta E_{b h}^{\prime}$ justifies our initial assumption that the local temperature is $T^{\prime}$.

We see that at the matching point the entropy of the black hole (3.1) is carried by 1-5 strings, the contributions from 1-1 and 5-5 strings are suppressed by a factor of $\frac{1}{\cosh ^{4} \alpha_{\min }}$. This depends crucially on the deep throat geometry outside the horizon. This result is consistent with that from precise counting [9] and resolves a puzzle in [4]. It was thought in [4] that at the matching point the metric (3.1) could be trusted all the way to the horizon and therefore a much larger gravitational field dressing would exist. The local temperature would therefore always be of order the string scale and it was puzzling why the 1-1 and 5-5 strings did not contribute to the total entropy. Indeed under this assumption the calculation shows that the entropy from 1-1 and 5-5 strings are of the same order as 1-5 strings (and they are all of the same order of the black hole entropy). Now we see that the gravitational dressing effect actually stops at $r \sim r^{*}$ so that the local temperature is small in string units and the entropy contributions from 1-1 and 5-5 strings are suppressed. 


\subsection{Six Dimensional Black Holes with D 0- and D 4-brane Charges}

The string metric of the six dimensional black hole with D 0- and D 4-brane charges is given by

$$
\begin{aligned}
d s^{2}= & \left(H_{0} H_{4}\right)^{1 / 2}\left[-\left(H_{0} H_{4}\right)^{-1} f d t^{2}+\left(H_{4}\right)^{-1}\left(d y_{1}^{2}+d y_{2}^{2}+d y_{3}^{2}+d y_{4}^{2}\right)\right. \\
& \left.+f^{-1} d r^{2}+r^{2} d \Omega_{4}^{2}\right]
\end{aligned}
$$

where

$$
\begin{aligned}
H_{i} & =1+\frac{r_{0}^{3} \sinh ^{2} \alpha_{i}}{r^{3}} \quad(i=0,4) \\
f & =1-\frac{r_{0}^{3}}{r^{3}}
\end{aligned}
$$

The $y_{a}$ are coordinates on the internal $T^{4}$. The dilaton is $e^{2 \phi}=H_{0}^{3 / 2} H_{4}^{-1 / 2}$. This black hole is a solution to the type IIA string theory on $T^{4}$. The horizon is at $r=r_{0}$. The extremal limit of the black hole can be obtained by letting $r_{0} \rightarrow 0$ while fixing $r_{0}^{3} \sinh ^{2} \alpha_{i}$ and has zero horizon area. The extremal limit preserves $1 / 4$ of the supersymmetries.

The analysis and result of this black hole are similar to the previous one. The matching between the black hole and D-branes and strings occurs when

$$
r_{0} \sim \frac{\alpha^{1 / 2}}{\left(\cosh \alpha_{0} \cosh \alpha_{4}\right)^{1 / 2}}
$$

Let $\cosh \alpha_{\min }$ and $\cosh \alpha_{\max }$ be the smaller and larger one of $\cosh \alpha_{0}$ and $\cosh \alpha_{4}$. The spatial geometry outside the horizon is the "bottle neck": the radius of the $S_{4}$ first decreases, it reaches the minimum at $r \sim r_{0}\left(\cosh \alpha_{\text {min }}\right)^{2 / 3}$ and then increases. The stringy behavior smears out the region outside the horizon all the way to the point where the curvature becomes of order the string scale again. Unlike the black hole considered in 3.1, the gravitational field dressing will appear only when the hierarchy between $\cosh \alpha_{0}$ and $\cosh \alpha_{4}$ is large enough, more precisely only when $\cosh \alpha_{\max }>\cosh ^{3} \alpha_{\min }$.

Now let us consider the statistical description of the system. It should be described by $Q_{0} \mathrm{D}$ 0-branes smeared in the 4-torus and $Q_{4} \mathrm{D}$ 4-branes with some excitations. We will see that the open string gas picture gives consistent result. There are three kinds of open strings: $0-0,4-4$ and 0-4 strings. We assume they live at local temperature $T^{\prime}$, which is obtained from the Hawking temperature and calculate the entropy and energy carried by them. We will see that they agree with the black hole entropy and excess energy. The calculation has to be done locally. 
The 4-4 strings are a four dimensional gas. The entropy is given by

$$
S_{44} \sim Q_{4}^{2} T^{\prime 4} V^{\prime} \sim \begin{cases}\frac{S_{b h}}{\cosh ^{12} \alpha_{4}}, & \text { if } \cosh \alpha_{0}>\cosh ^{3} \alpha_{4} \\ \frac{S_{b h}}{\cosh ^{4} \alpha_{0}}, & \text { otherwise. }\end{cases}
$$

where $V^{\prime}$ is the local volume of the 4-torus and $S_{b h}$ is the black hole entropy at the matching point. The 0-0 strings are related to the 4-4 strings by T-dualizing all the internal directions in the 4-torus. To get $S_{00}$, we can simply "T-dualize" equation (3.15)

$$
S_{00} \sim \begin{cases}\frac{S_{b h}}{\cosh ^{12} \alpha_{0}}, & \text { if } \cosh \alpha_{4}>\cosh ^{3} \alpha_{0} \\ \frac{S_{b h}}{\cosh ^{4} \alpha_{4}}, & \text { otherwise. }\end{cases}
$$

The 0-4 strings have ND or DN boundary condition in the four internal directions. The ND or DN boundary condition does not change the number of species of the strings, so there are $Q_{0} Q_{4}$ of them. The entropy from the $0-4$ strings is

$$
S_{04} \sim Q_{0} Q_{4} \sim S_{b h}
$$

The total entropy $S_{\text {open }}$ and the total local energy $E_{\text {open }}^{\prime}$ carried by the 3 kinds of open strings is

$$
\begin{aligned}
& S_{\text {open }}=S_{00}+S_{44}+S_{04} \sim S_{04} \sim S_{b h} \\
& E_{\text {open }}^{\prime} \sim T^{\prime} S_{\text {open }} \sim \Delta E_{b h}^{\prime}
\end{aligned}
$$

where $\Delta E_{b h}^{\prime}$ is the local black hole excess energy.

So we see that at the matching point, the entropy of the black hole (3.12) is carried by 0-4 strings, the contributions from 0-0 and 4-4 strings are suppressed by some powers in $\cosh \alpha_{i}(i=0,4)$.

\subsection{Four Dimensional Black Holes with D 2-brane, D 6-brane and NS 5-brane Charges}

The string metric of the four dimensional black hole with D 2-brane, D 6-brane and NS 5-brane charges is given by

$$
\begin{aligned}
d s^{2} & =\left(H_{2} H_{6}\right)^{1 / 2} H_{5}\left[-\left(H_{2} H_{6} H_{5}\right)^{-1} f d t^{2}+\left(H_{2} H_{6}\right)^{-1} d y_{1}^{2}+\left(H_{2} H_{6} H_{5}\right)^{-1} d y_{2}^{2}\right. \\
& \left.+\left(H_{6} H_{5}\right)^{-1}\left(d y_{3}^{2}+d y_{4}^{2}+d y_{5}^{2}+d y_{6}^{2}\right)+f^{-1} d r^{2}+r^{2} d \Omega_{2}^{2}\right]
\end{aligned}
$$

where

$$
\begin{aligned}
H_{i} & =1+\frac{r_{0} \sinh ^{2} \alpha_{i}}{r} \quad(i=2,5,6) \\
f & =1-\frac{r_{0}}{r}
\end{aligned}
$$


The $y_{a}$ are coordinates on the internal $T^{6}$ which are identified with $L_{a}$. The dilaton is $e^{2 \phi}=H_{2}^{1 / 2} H_{6}^{-3 / 2} H_{5}$. This black hole is a solution to the type IIA string theory on $T^{6}$. The horizon is at $r=r_{0}$. The extremal limit of the black hole can be obtained by letting $r_{0} \rightarrow 0$ while fixing $r_{0} \sinh ^{2} \alpha_{i}$ and has zero horizon area. The extremal limit preserves $1 / 8$ of the supersymmetries. If we set the 5 -brane charge $Q_{5}=0$, the black hole carries only the two RR charges, for convenience this case will be included in the discussion of section 4. In this section, we consider the case when all charges are large.

The analysis and result for this black hole are similar to the previous two black holes. The matching between the black hole and D-branes, NS 5-branes and strings occurs at

$$
r_{0} \sim \frac{\alpha^{\prime 1 / 2}}{\left(\cosh \alpha_{2} \cosh \alpha_{6}\right)^{1 / 2} \cosh \alpha_{5}}
$$

Let $\cosh \alpha_{\min }$ and $\cosh \alpha_{\max }$ be the smallest and the largest of $\cosh \alpha_{i}(i=2,5,6)$. The spatial geometry outside the horizon has a deep throat from $r_{0}$ to $r_{0} \cosh ^{2} \alpha_{\text {min }}$. Note this will not happen if we set $Q_{5}=0$. Adding NS 5-branes balances the dilaton and gives us the deep throat. The whole throat is smeared out by stringy behavior at the matching point. If all $\cosh \alpha_{i}$ are of the same order, there is no large gravitational field dressing; if $\cosh \alpha_{\max }>>\cosh \alpha_{\min }$, then there is still a large dressing.

Now let us consider the statistical description of the system. It should be described by $Q_{2} \mathrm{D}$ 2-branes, $Q_{6} \mathrm{D}$ 6-branes and $Q_{5}$ NS 5-branes with some excitations. The brane configuration is as follows:

$$
\begin{array}{ccccccc}
D 2: 1 & 2 & & & & \\
D 6: 1 & 2 & 3 & 4 & 5 & 6 \\
N S 5: & 2 & 3 & 4 & 5 & 6
\end{array}
$$

We will see that the open string gas picture gives consistent result. There are three kinds of open strings: 2-2, 6-6 and 2-6 strings. We assume they live at local temperature $T^{\prime}$, which is obtained from the Hawking temperature and calculate the entropy and energy carried by them. We will see that they agree with the black hole entropy and excess energy.

Before we do the calculation, we have to take care of the new feature brought in by the NS 5-branes. The 5-branes are uniformly distributed in the $y_{1}$ direction. They cut the 6-torus into $Q_{5}$ segments and each D 2- and D 6-brane into $Q_{5}$ pieces [10]. In each segment the $Q_{2}$ D-2 branes or $Q_{6}$ D 6-branes can not be wrapped around the $y_{1}$ direction. We need to know the local separation between adjacent 5 -branes to see whether at temperature $T^{\prime}$ 
the open strings can see this dimension or not. Let $L_{1}$ and $L_{1}^{\prime}$ be the asymptotic and local size of the $y_{1}$ circle. We have

$$
Q_{5} \sim \frac{L_{1} r_{0} \sinh 2 \alpha_{5}}{\alpha^{\prime}} \sim \frac{L_{1} \cosh \alpha_{5}}{\alpha^{1 / 2}\left(\cosh \alpha_{2} \cosh \alpha_{6}\right)^{1 / 2}} \sim \frac{L_{1}^{\prime}}{\alpha^{1 / 2}}
$$

where the second step follows from (3.21) and the relation between $L_{1}$ and $L_{1}^{\prime}$ is independent of whether there is large dressing or not. So the separation between adjacent 5 -branes is always of order $\alpha^{1 / 2}$. On the other hand, the local temperature $T^{\prime}$ is always much smaller than the string scale. So, the $y_{2}$ direction is frozen for the open strings: the 2-6 and 2-2 strings become an one dimensional gas, the 6-6 strings become a five dimensional gas.

The entropy from the 6-6 strings is the sum of the contributions from each of the $Q_{5}$ segments

$$
S_{66} \sim Q_{5} Q_{6}^{2} T^{\prime 5} L_{2}^{\prime} L_{3}^{\prime} L_{4}^{\prime} L_{5}^{\prime} L_{6}^{\prime} \sim \frac{S_{b h}}{\cosh ^{4} \alpha_{\min }}
$$

where $L_{i}^{\prime}(i=2,3,4,5,6)$ are the local sizes of the $y_{i}$ circles and $S_{b h}$ is the black hole entropy at the matching point. The 2-2 strings are related to the 6-6 strings by T-dualizing the $y_{2}, y_{3}, y_{4}, y_{5}$ directions. Since (3.24) is invariant under this operation, we must have $S_{22} \sim S_{66}$. The entropy from the $2-6$ strings is

$$
S_{26} \sim Q_{5} Q_{2} Q_{6} L_{2}^{\prime} T^{\prime} \sim S_{b h}
$$

The total entropy $S_{\text {open }}$ and local energy $E_{\text {open }}^{\prime}$ carried by the three kinds of open strings are

$$
\begin{aligned}
& S_{\text {open }}=S_{22}+S_{66}+S_{26} \sim S_{26} \sim S_{b h} \\
& E_{\text {open }}^{\prime} \sim T^{\prime} S_{\text {open }} \sim \Delta E_{b h}^{\prime}
\end{aligned}
$$

where $\Delta E_{b h}^{\prime}$ is the local black hole excess energy.

So we see that at the matching point, the entropy of the black hole (3.19) is carried by 2-6 strings, the contributions from 2-2 and 6-6 strings are suppressed by a factor of $\frac{1}{\cosh ^{4} \alpha_{\text {min }}}$. This is consistent with the result from precise counting 111.

\section{Four Dimensional Black Hole with 4 RR Charges}

In this section we consider a four dimensional black hole that carries $4 \mathrm{RR}$ charges. We will go through the calculation in some detail because it differs from black holes considered 
in section 3 in certain aspects. The string metric of the black hole is

$$
\begin{aligned}
d s^{2}= & \left(H_{1} H_{2} H_{3} H_{4}\right)^{1 / 2}\left[-\left(H_{1} H_{2} H_{3} H_{4}\right)^{-1} f d t^{2}+\left(H_{1} H_{3}\right)^{-1} d y_{1}^{2}+\left(H_{1} H_{4}\right)^{-1} d y_{2}^{2}\right. \\
& +\left(H_{1} H_{2}\right)^{-1} d y_{3}^{2}+\left(H_{2} H_{4}\right)^{-1} d y_{4}^{2}+\left(H_{2} H_{3}\right)^{-1} d y_{5}^{2}+\left(H_{3} H_{4}\right)^{-1} d y_{6}^{2} \\
& \left.+f^{-1} d r^{2}+r^{2} d \Omega_{2}^{2}\right]
\end{aligned}
$$

where

$$
\begin{aligned}
H_{i} & =1+\frac{r_{0} \sinh ^{2} \alpha_{i}}{r} \quad(i=1,2,3,4) \\
f & =1-\frac{r_{0}}{r}
\end{aligned}
$$

The $y_{a}(a=1,2,3,4,5,6)$ are coordinates on the internal $T^{6}$ which are identified with length $L_{a}$. The dilaton is a constant. This black hole is a solution to the type IIB string theory on $T^{6}$. The event horizon is at $r=r_{0}$. The extremal limit of the black hole can be obtained by letting $r_{0} \rightarrow 0$ while fixing $r_{0} \sinh ^{2} \alpha_{i}$ and has nonvanishing horizon area if all four charges are nonzero. The extremal limit preserves $1 / 8$ of the supersymmetries and can be thought of as a superposition of four different kinds of extremal black 3-branes that are extended in the $(123),(345),(561),(246)$ directions separately and smeared out in the rest directions. For the rest of this section, we will consider the case where at least two charges are large.

The energy, excess energy, entropy, Hawking temperature and four RR charges of the black hole are

$$
\begin{aligned}
E & \sim \frac{L_{1} L_{2} L_{3} L_{4} L_{5} L_{6}}{g^{2} \alpha^{\prime 4}} r_{0}\left(\cosh 2 \alpha_{1}+\cosh 2 \alpha_{2}+\cosh 2 \alpha_{3}+\cosh 2 \alpha_{4}\right) \\
\Delta E_{b h} & \sim \frac{L_{1} L_{2} L_{3} L_{4} L_{5} L_{6}}{g^{2} \alpha^{\prime 4}} r_{0}\left(\frac{1}{\cosh ^{2} \alpha_{1}}+\frac{1}{\cosh ^{2} \alpha_{2}}+\frac{1}{\cosh ^{2} \alpha_{3}}+\frac{1}{\cosh ^{2} \alpha_{4}}\right) \\
S_{b h} & \sim \frac{L_{1} L_{2} L_{3} L_{4} L_{5} L_{6}}{g^{2} \alpha^{\prime 4}} r_{0}^{2} \cosh \alpha_{1} \cosh \alpha_{2} \cosh \alpha_{3} \cosh \alpha_{4} \\
T & \sim \frac{1}{r_{0} \cosh \alpha_{1} \cosh \alpha_{2} \cosh \alpha_{3} \cosh \alpha_{4}} \\
Q_{1} & \sim \frac{L_{4} L_{5} L_{6}}{g \alpha^{\prime 2}} r_{0} \sinh 2 \alpha_{1} \\
Q_{2} & \sim \frac{L_{1} L_{2} L_{6}}{g \alpha^{\prime 2}} r_{0} \sinh 2 \alpha_{2} \\
Q_{3} & \sim \frac{L_{2} L_{3} L_{4}}{g \alpha^{\prime 2}} r_{0} \sinh 2 \alpha_{3} \\
Q_{4} & \sim \frac{L_{1} L_{3} L_{5}}{g \alpha^{\prime 2}} r_{0} \sinh 2 \alpha_{4}
\end{aligned}
$$


where to obtain $\Delta E_{b h}$ we have used the relation that $\cosh 2 \alpha_{i}-\sinh 2 \alpha_{i} \sim \frac{1}{\cosh ^{2} \alpha_{i}}$ for any value of $\alpha_{i}$.

The matching point is

$$
r_{0} \sim \frac{\alpha^{1 / 2}}{\left(\cosh \alpha_{1} \cosh \alpha_{2} \cosh \alpha_{3} \cosh \alpha_{4}\right)^{1 / 2}}
$$

At the matching point, the system should be described by the corresponding four kinds of D 3-branes with some excitations. The D3-branes are wrapped around the internal $T^{6}$ in the following way:

$$
\begin{array}{lllllll}
(D 3)_{1}: 1 & 2 & 3 & & & \\
(D 3)_{2}: & & 3 & 4 & 5 & \\
(D 3)_{3}: 1 & & & & 5 & 6 \\
(D 3)_{4}: & 2 & & 4 & & 6
\end{array}
$$

There is a qualitative difference between the case where two or three charges are large and the case where all four charges are large. Let us discuss these two different cases in the next two subsections separately.

\subsection{The Black Hole with Two or Three Large Charges}

Without losing generality, let us set $\alpha_{4}=0$, so $Q_{4}=0$ and the black hole carries $Q_{1}, Q_{2}, Q_{3}$ only. The D-branes have a large gravitational field dressing and the metric at the the D-branes (i.e., $r \sim r_{0}$ ) is

$$
\begin{aligned}
d s^{2} \sim & \left(\cosh \alpha_{1} \cosh \alpha_{2} \cosh \alpha_{3}\right)\left[-\frac{d t^{2}}{\cosh ^{2} \alpha_{1} \cosh ^{2} \alpha_{2} \cosh ^{2} \alpha_{3}}+\frac{d y_{1}^{2}}{\cosh ^{2} \alpha_{1} \cosh ^{2} \alpha_{3}}\right. \\
& +\frac{d y_{2}^{2}}{\cosh ^{2} \alpha_{1}}+\frac{d y_{3}^{2}}{\cosh ^{2} \alpha_{1} \cosh ^{2} \alpha_{2}}+\frac{d y_{4}^{2}}{\cosh ^{2} \alpha_{2}}+\frac{d y_{5}^{2}}{\cosh ^{2} \alpha_{2} \cosh ^{2} \alpha_{3}}+\frac{d y_{6}^{2}}{\cosh ^{2} \alpha_{3}} \\
& \left.+\frac{\alpha^{\prime} d \Omega_{2}^{2}}{\cosh \alpha_{1} \cosh \alpha_{2} \cosh \alpha_{3}}\right]
\end{aligned}
$$

where we have substituted (4.4) into the metric. Let us use $T^{\prime}, \Delta E_{b h}^{\prime}$ to denote the local temperature and local energy, then

$$
\begin{aligned}
T^{\prime} & \sim \frac{1}{\alpha^{1 / 2}} \\
\Delta E_{b h}^{\prime} & \sim \frac{L_{1} L_{2} L_{3} L_{4} L_{5} L_{6}}{g^{2} \alpha^{\prime / 2}}
\end{aligned}
$$


We will assume that the excitations are described by the massless open strings and see that this gives consistent result. There are six kinds of open strings in general: three of them end on the same kind of D-branes, the rest three stretch between different kinds of D-branes. We assume the local temperature to be $\frac{1}{\alpha^{\prime 1 / 2}}$ and calculate the entropy and energy carried by them. We will see that they agree with the black hole entropy and excess energy.

Let us use $i$ - $j$ open strings to denote the open strings stretched between the $i$ th and $j$ th kind (which are labeled in (4.5)) of D 3-branes and $S_{i j}$ to denote the entropy contribution from them. There is an obvious symmetry among the three kinds of D 3-branes. It is enough to just calculate $S_{11}$ and $S_{12}$, the rest can be obtained by the symmetry.

We consider $S_{11}$ first. The 1-1 open strings form a three dimensional gas. The $Q_{1} \mathrm{D}$ 3 -branes are evenly distributed in the $y_{4}, y_{5}, y_{6}$ directions. So there are $Q_{1}^{2} \frac{\alpha^{\prime 3 / 2}}{L_{4}^{\prime} L_{5}^{\prime} L_{6}^{\prime}}$ species of open strings, for the same reason explained in section 3.1. The entropy from the 1-1 strings is

$$
S_{11} \sim Q_{1}^{2} \frac{\alpha^{\prime 3 / 2}}{L_{4}^{\prime} L_{5}^{\prime} L_{6}^{\prime}} T^{\prime 3} L_{1}^{\prime} L_{2}^{\prime} L_{3}^{\prime} \sim S_{b h} \tanh ^{2} \alpha_{1}
$$

where

$$
S_{b h} \sim \frac{L_{1} L_{2} L_{3} L_{4} L_{5} L_{6}}{g^{2} \alpha^{\prime 3}}
$$

is the black hole entropy at the matching point, which follows from (4.3) and (4.4).

Next we consider the 1-2 open strings. The 1-2 open strings have NN(NeumannNeumann) boundary condition in the $y_{3}$ direction, DD(Dirichlet-Dirichlet) boundary condition in the $y_{6}$ direction and ND or DN boundary condition in the $y_{1}, y_{2}, y_{4}, y_{5}$ directions. It is a one dimensional gas. The ND or DN boundary condition does not change the number of species of open strings. The DD boundary condition in the $y_{6}$ direction gives a factor of $\frac{\alpha^{\prime 1 / 2}}{L_{6}^{\prime}}$ to the number of species for the same reason. The entropy from the 1-2 strings is

$$
S_{12} \sim Q_{1} Q_{2} \frac{\alpha^{1 / 2}}{L_{6}^{\prime}} T^{\prime} L_{3}^{\prime} \sim S_{b h} \tanh \alpha_{1} \tanh \alpha_{2}
$$

Because of the symmetry among the three kinds of D-branes, it is clear that the entropy from the $i$ - $j$ open strings is

$$
S_{i j} \sim S_{b h} \tanh \alpha_{i} \tanh \alpha_{j}
$$


The total entropy $S_{\text {open }}$ and local energy $E_{\text {open }}^{\prime}$ carried by these open strings are

$$
\begin{aligned}
& S_{\text {open }}=\sum_{i, j=1}^{3} S_{i j} \sim S_{b h} \sum_{i, j=1}^{3} \tanh \alpha_{i} \tanh \alpha_{j} \sim S_{b h} \\
& E_{\text {open }}^{\prime} \sim T^{\prime} S_{\text {open }} \sim \Delta E_{b h}^{\prime}
\end{aligned}
$$

The sum $\sum_{i, j=1}^{3} \tanh \alpha_{i} \tanh \alpha_{j}$ in the entropy expression includes six terms and takes value between 3 and 6 since we have assumed at least two charges are large.

Now let us briefly discuss the two large charges case and three large charges case separately. If only two charges are large, we can ignore the third charge. We have two different kinds of D 3-branes that intersect at a line, which is T-dual to the system of 0-branes and 4-branes smeared out in two more dimensions or the system of 1-branes and 5 -branes smeared out in one more dimension or the system of 2-branes and 6-branes. Up to T-duality, this black hole and the black holes considered in section 3.1 and 3.2 are all the possible black holes that belong to category (B) that we classified in the introduction. The correspondence principle can be applied to all of them.

If three charges are large, the entropy can also be accounted for by massless open strings. However, we have to remember that the precise counting of entropy has already been done for a four dimensional near extremal black holes with three large charges [11], the entropy was reproduced at weak coupling in the limit that the excitations of the 4th kind of charge are much lighter than the other three charges and form a dilute gas. One might expect that similar precise counting can be done for this black hole. This would suggest that the entropy is carried by brane-antibrane excitations rather than open strings at the matching point. We will do such a calculation in section 4.2. The result is that the entropy contribution from the 4th kind of branes and antibranes is of the same order as that from the gas of the open strings. But the gas of the brane-antibrane pairs is not dilute and the calculation depends on the assumption that the 4th kind of branes and antibranes make up the entropy with the other three kinds of branes independently. If this assumption is correct, then a complete analysis would include both types of excitations. Which form the excitations really take should depend on which type gives the larger entropy.

\subsection{The Black Hole with 4 Large RR Charges}

One might wonder since the entropy of four dimensional extremal black hole with four charges has been counted precisely [10] 112], why we are still interested in this case. If the 
black hole is exactly at the extremal limit, then there is nothing new that needs to be said. If the black hole is only near extremal with four large charges, we can still apply the correspondence principle to it and ask what the description is at the matching point.

Let $r^{*}=r_{0} \cosh ^{2} \alpha_{\text {min }}$, where $\cosh \alpha_{\text {min }}$ is the smallest of $\cosh \alpha_{i}(i=1,2,3,4)$. The spatial geometry outside the horizon has a deep throat from $r_{0}$ to $r^{*}$. We see the crucial difference between this case and the previous case: turning on the 4th charge causes the development of the deep throat, the length of the throat is determined by the smallest charge (by $\cosh \alpha_{\min }$, strictly speaking). The whole throat is smeared out by stringy behavior at the matching point. If all $\cosh \alpha_{i}$ are of the same order there is no large gravitational field dressing; if there is a hierarchy between the $\cosh \alpha_{i}$ 's, then there is still a large dressing. In either case, we can write $H_{i}\left(r=r^{*}\right) \sim \frac{\cosh ^{2} \alpha_{i}}{\cosh ^{2} \alpha_{m i n}}$. In the following, we will treat both cases together.

The metric at the D-branes (i.e., $r \sim r^{*}$ ) is

$$
\begin{aligned}
& d s^{2} \sim\left(\frac{\cosh \alpha_{1} \cosh \alpha_{2} \cosh \alpha_{3} \cosh \alpha_{4}}{\cosh ^{4} \alpha_{\min }}\right)\left[-\frac{d t^{2}}{\left(\frac{\cosh \alpha_{1} \cosh \alpha_{2} \cosh \alpha_{3} \cosh \alpha_{4}}{\cosh ^{4} \alpha_{m i n}}\right)^{2}}\right. \\
& +\frac{d y_{1}^{2}}{\left(\frac{\cosh \alpha_{1} \cosh \alpha_{3}}{\cosh ^{2} \alpha_{\min }}\right)^{2}}+\frac{d y_{2}^{2}}{\left(\frac{\cosh \alpha_{1} \cosh \alpha_{4}}{\cosh ^{2} \alpha_{\min }}\right)^{2}}+\frac{d y_{3}^{2}}{\left(\frac{\cosh \alpha_{1} \cosh \alpha_{2}}{\cosh ^{2} \alpha_{\min }}\right)^{2}}+\frac{d y_{4}^{2}}{\left(\frac{\cosh \alpha_{2} \cosh \alpha_{4}}{\cosh ^{2} \alpha_{m i n}}\right)^{2}} \\
& \left.+\frac{d y_{5}^{2}}{\left(\frac{\cosh \alpha_{2} \cosh \alpha_{3}}{\cosh ^{2} \alpha_{\min }}\right)^{2}}+\frac{d y_{6}^{2}}{\left(\frac{\cosh \alpha_{3} \cosh \alpha_{4}}{\cosh ^{2} \alpha_{\min }}\right)^{2}}+\frac{\alpha^{\prime} d \Omega_{2}^{2}}{\left(\frac{\cosh \alpha_{1} \cosh \alpha_{2} \cosh \alpha_{3} \cosh \alpha_{4}}{\cosh ^{4} \alpha_{\min }}\right)}\right]
\end{aligned}
$$

It approaches the metric (4.6) smoothly as $\alpha_{\min } \rightarrow 0$.

Independent of whether there is large dressing or not, the local temperature and local excess energy are always given by

$$
\begin{aligned}
T^{\prime} & \sim \frac{1}{\alpha^{\prime 1 / 2} \cosh ^{2} \alpha_{\min }} \\
\Delta E_{b h}^{\prime} & \sim \frac{L_{1} L_{2} L_{3} L_{4} L_{5} L_{6}}{g^{2} \alpha^{\prime 7 / 2} \cosh ^{4} \alpha_{\min }}
\end{aligned}
$$

Compared with the black hole carrying less than four large charges, $T^{\prime}$ and $\Delta E_{b h}^{\prime}$ are suppressed by some power in $\frac{1}{\cosh \alpha_{\min }}$.

Another special feature about the four large charges case is that most of the entropy is the BPS entropy. We can imagine obtaining such a black hole by adding excess energy to an extremal black hole with exactly the same charges (incidentally we notice that if we 
add or take away some excess energy from the system at the matching point, it will stay at the matching point, which does not happen for the less than four large charges case). The entropy of the extremal black hole is what we call the BPS entropy and is given by

$$
S_{e x}=2 \pi \sqrt{Q_{1} Q_{2} Q_{3} Q_{4}}
$$

The excess entropy $\Delta S_{b h}$ is defined to be the difference between the total entropy and the BPS entropy

$$
\begin{aligned}
\Delta S_{b h} \equiv S_{b h}-S_{e x} & =S_{b h}\left(1-\sqrt{\tanh \alpha_{1} \tanh \alpha_{2} \tanh \alpha_{3} \tanh \alpha_{4}}\right) \\
& \sim \frac{S_{b h}}{\cosh ^{2} \alpha_{\text {min }}}
\end{aligned}
$$

where we have used the property $1-\tanh \alpha_{i} \sim \frac{1}{\cosh ^{2} \alpha_{i}}$ for $\cosh \alpha_{i}>>1$. At the matching point (or in the weak coupling limit for that matter), the BPS entropy comes from the four kinds of D-branes [12] and can be counted precisely. So what needs to be reproduced is the excess entropy $\Delta S_{b h}$.

We will start by considering the open string gas picture and see that it fails to work. We then turn to the discussion of the brane-antibrane picture (i.e., the excitations take the form of brane and antibrane pairs), which is a possible resolution.

We will assume the local temperature to be $T^{\prime}$ and calculate the entropy and energy carried by the massless open strings. There are ten different kinds of open strings: four of them end on the same kind of D-branes, the rest six stretch between two different kinds of D-branes.

We consider $S_{11}$ first. The 1-1 open strings should now have length of order $\frac{\alpha^{\prime 1 / 2}}{\cosh ^{2} \alpha_{m i n}}$. The number of species of 1-1 strings is $Q_{1}^{2} \frac{\alpha^{\prime 3 / 2}}{L_{4}^{\prime} L_{5}^{\prime} L_{6}^{\prime} \cosh ^{6} \alpha_{\min }}$ and the entropy is

$$
S_{11} \sim Q_{1}^{2} \frac{\alpha^{\prime 3 / 2}}{L_{4}^{\prime} L_{5}^{\prime} L_{6}^{\prime} \cosh ^{6} \alpha_{\min }} T^{\prime 3} L_{1}^{\prime} L_{2}^{\prime} L_{3}^{\prime} \sim \frac{S_{b h}}{\cosh ^{12} \alpha_{m i n}}
$$

Next, we consider $S_{12}$. The number of species of $1-2$ strings is now $Q_{1} Q_{2} \frac{\alpha^{1 / 2}}{L_{6}^{\prime} \cosh ^{2} \alpha_{m i n}}$. The entropy from the 1-2 strings is

$$
S_{12} \sim Q_{1} Q_{2} \frac{\alpha^{\prime 1 / 2}}{L_{6}^{\prime} \cosh ^{2} \alpha_{\min }} T^{\prime} L_{3}^{\prime} \sim \frac{S_{b h}}{\cosh ^{4} \alpha_{\min }}
$$

Because of the symmetry among the 4 kinds of D-branes, it is clear that the entropy from the $i$ - $j$ open strings is

$$
\begin{aligned}
S_{i i} & \sim \frac{S_{b h}}{\cosh ^{12} \alpha_{\min }}(i=1,2,3,4) \\
S_{i j} & \sim \frac{S_{b h}}{\cosh ^{4} \alpha_{\min }}(i, j=1,2,3,4, i \neq j)
\end{aligned}
$$


The total entropy and local energy are

$$
\begin{aligned}
& S_{\text {open }}=\sum_{i, j=1}^{4} S_{i j} \sim \frac{S_{b h}}{\cosh ^{4} \alpha_{\text {min }}} \\
& E_{\text {open }}^{\prime} \sim T^{\prime} S_{\text {open }} \sim \frac{L_{1} L_{2} L_{3} L_{4} L_{5} L_{6}}{g^{2} \alpha^{\prime / 2} \cosh ^{6} \alpha_{\text {min }}}
\end{aligned}
$$

They are much smaller than the $\Delta S_{b h}$ and $\Delta E_{b h}^{\prime}$. We can also do the alternative calculation by putting all the excess energy $\Delta E_{b h}^{\prime}$ into the open strings. This leads to a local temperature of order $\frac{1}{\alpha^{\prime 1 / 2}\left(\cosh \alpha_{4}\right)^{4 / 3}}$, higher than $T^{\prime}$ and an entropy of order $\frac{S_{b h}}{\left(\cosh \alpha_{4}\right)^{8 / 3}}$, still much smaller than $\Delta S_{b h}$.

This shows that the open string gas picture does not work. If the correspondence principle can be applied to this case in the usual context, we need to search for other forms of excitations. In the following, we will consider the brane-antibrane picture.

We consider the problem in the framework of canonical ensemble. The D-brane system is immersed in temperature $T^{\prime}$. Let us say creating a certain number of brane-antibrane pairs costs local energy $E_{\text {pair }}^{\prime}$ and increases the entropy by $S_{\text {pair }}$. The increase in the energy gives a Boltzman factor $e^{-\frac{E_{\text {pair }}^{\prime}}{T^{\prime}}}$, while the increase in the entropy means the degeneracy of microstates is increased by $e^{S_{\text {pair }}}$ times. To find out how many pairs are excited, we need to extremize $e^{-\frac{E_{\text {pair }}^{\prime}}{T^{\prime}}+S_{\text {pair }}}$ with respect to the number of pairs.

Since the system is near BPS, we can extremize with respect to each kind of branes separately. Let us focus on the first kind of D-brane and assume $Q_{\overline{1}}$ pairs are excited. We assume $S_{\text {pair }}$ is given by

$$
S_{\text {pair }} \sim \sqrt{Q_{\overline{1}} Q_{2} Q_{3} Q_{4}}
$$

, i.e., the $Q_{1}+Q_{\overline{1}}$ branes and $Q_{\overline{1}}$ antibranes make up the entropy with the other three kinds of branes independently. The $E_{\text {pair }}^{\prime}$ is given by

$$
E_{\text {pair }}^{\prime} \sim Q_{\overline{1}} \frac{L_{1}^{\prime} L_{2}^{\prime} L_{3}^{\prime}}{g \alpha^{\prime 2}}
$$

Using (4.3), (4.4) and (4.13) to extremize $e^{-\frac{E_{p a i r}^{\prime}}{T^{\prime}}+S_{\text {pair }}}$ with respect to $Q_{\overline{1}}$, we find

$$
\frac{Q_{\overline{1}}}{Q_{1}} \sim \frac{1}{\cosh ^{4} \alpha_{\min }}
$$

Because of the symmetry among the 4 kinds of D-branes, we must also have

$$
\frac{Q_{\overline{2}}}{Q_{2}} \sim \frac{Q_{\overline{3}}}{Q_{3}} \sim \frac{Q_{\overline{4}}}{Q_{4}} \sim \frac{1}{\cosh ^{4} \alpha_{\min }}
$$


It is easy to confirm that the entropy and excess energy contributions from each kind of D-branes are of the same order and the total entropy and excess energy are

$$
\begin{aligned}
& S_{\text {pair }} \sim \frac{S_{b h}}{\cosh ^{2} \alpha_{\text {min }}} \sim \Delta S_{b h} \\
& E_{\text {pair }}^{\prime} \sim \Delta E_{b h}^{\prime}
\end{aligned}
$$

We are using the same symbol $S_{\text {pair }}$ and $E_{\text {pair }}^{\prime}$ here, hopefully this will not cause any confusion.

This calculation depends critically on the assumption (4.21). It was first shown in 13] that for black holes with multiple charges, one can formally define brane and antibrane numbers for each charge and write the energy, entropy, etc., in terms of the brane and antibrane numbers. Take the black hole that we are considering as a example, the entropy can be written as

$$
S=2 \pi\left(\sqrt{Q_{1}}+\sqrt{Q_{\overline{1}}}\right)\left(\sqrt{Q_{2}}+\sqrt{Q_{\overline{2}}}\right)\left(\sqrt{Q_{3}}+\sqrt{Q_{\overline{3}}}\right)\left(\sqrt{Q_{4}}+\sqrt{Q_{\overline{4}}}\right)
$$

Moreover, if one fixes the charges and the excess energy of the black hole, assumes the entropy is given by (1.26) and maximizes the entropy, one finds the antibrane numbers to be the same as obtained from the black hole. What we have done is not exactly the same in that it is not a asymptotic calculation but a local calculation. It is interesting to see that it gives the right result.

We can do the same calculation for the three large charges case considered in 4.1. The number of pairs of the 4th kind of D-branes excited is

$$
Q_{\overline{4}} \sim \frac{L_{1} L_{3} L_{5}}{g \alpha^{\prime 3 / 2}\left(\cosh \alpha_{1} \cosh \alpha_{2} \cosh \alpha_{3}\right)^{1 / 2}}
$$

and the entropy from them is

$$
S_{\text {pair }} \sim \sqrt{Q_{1} Q_{2} Q_{3} Q_{\overline{4}}} \sim S_{b h}
$$

We can estimate the local density of these branes and antibranes in the $y_{1} y_{3} y_{5}$ volume in the internal torus. It turns out to be of order $\frac{1}{g \alpha^{\prime 3 / 2}}$, so it is not a dilute gas.

\section{Eight Dimensional Black Hole with Two RR Charges}

In this section, we consider an eight dimensional near extremal black hole that carries two RR charges. Unlike the black holes considered in the previous sections, the extremal 
limit of this black hole is what is called non-marginal bound state. For a discussion of this kind of black holes, see [14]. The string metric of the black hole is

$$
d s^{2}=H^{1 / 2}\left[-H^{-1} f d t^{2}+\left(1+(H-1) \cos ^{2} \theta\right)^{-1}\left(d y_{1}^{2}+d y_{2}^{2}\right)+f^{-1} d r^{2}+r^{2} d \Omega_{6}^{2}\right]
$$

where

$$
\begin{aligned}
& H=1+\frac{r_{0}^{5} \sinh ^{2} \alpha}{r^{5}} \\
& f=1-\frac{r_{0}^{5}}{r^{5}}
\end{aligned}
$$

The dilaton is $e^{2 \phi}=H^{3 / 2}\left(1+(H-1) \cos ^{2} \theta\right)^{-1}$. The $\theta$ is a constant angle whose physical meaning will become clear. The $y_{1}, y_{2}$ are coordinates on the internal $T^{2}$.

This black hole is a solution to the type IIA string theory on $T^{2}$. The extremal limit of this black hole was obtained in [15]. The non-extremal solution can be constructed along the same line. The horizon is at $r=r_{0}$. In the metric (5.1), the extremal limit can be obtained by letting $r_{0} \rightarrow 0$ while fixing $r_{0}^{5} \sinh ^{2} \alpha$ and it preserves $1 / 2$ of the supersymmetries. If $\cos ^{2} \theta=0$, the solution corresponds to $\mathrm{D} 0$-branes smeared out in the $y_{1}, y_{2}$ directions; if $\cos ^{2} \theta=1$, the solution corresponds to $\mathrm{D} 2$-branes extended in the $y_{1}, y_{2}$ directions; if $0<\cos ^{2} \theta<1$, the solution interpolates between the two limiting cases and carries both 0 -brane charge $(\propto \sin \theta)$ and 2-brane charge $(\propto \cos \theta)$.

To make the symmetry between the two charges more explicitly and facilitate the calculation, it is better to consider the T-dual picture in type IIB. Let us T-dualize along the $y_{2}$ direction and still use $y_{2}$ as the coordinate, the solution becomes

$$
\begin{aligned}
d s^{2}= & H^{1 / 2}\left[-H^{-1} f d t^{2}+\left(H^{-1} \cos ^{2} \theta+\sin ^{2} \theta\right) d y_{1}^{2}+\left(H^{-1} \sin ^{2} \theta+\cos ^{2} \theta\right) d y_{2}^{2}\right. \\
& \left.+2 \sin \theta \cos \theta\left(H^{-1}-1\right) d y_{1} d y_{2}+f^{-1} d r^{2}+r^{2} d \Omega_{6}^{2}\right]
\end{aligned}
$$

The dilaton is $e^{2 \phi}=H$. The $y_{1}, y_{2}$ are identified with length $L_{1}, L_{2}$. The metric looks a little bit complicated, however, if we perform a rotation in the $y_{1}-y_{2}$ plane:

$$
\left(\begin{array}{l}
y_{1} \\
y_{2}
\end{array}\right)=\left(\begin{array}{cc}
\cos \theta & -\sin \theta \\
\sin \theta & \cos \theta
\end{array}\right)\left(\begin{array}{l}
\tilde{y}_{1} \\
\tilde{y}_{2}
\end{array}\right)
$$

The metric becomes

$$
d s^{2}=H^{1 / 2}\left[-H^{-1} f d t^{2}+H^{-1} d \tilde{y}_{1}^{2}+d \tilde{y}_{2}^{2}+f^{-1} d r^{2}+r^{2} d \Omega_{6}^{2}\right]
$$

which we recognize as the non-extremal black string solution in IIB that carries RR two form electric charge smeared out in the $\tilde{y}_{2}$ direction. Note that (5.3) and (5.5) are physically 
different black holes: the rotation does not make any difference locally in the $y_{1}-y_{2}$ plane, but the global coordinate identifications of the 2-planes are different.

Now let us focus on solution (5.3). The energy, excess energy, entropy, Hawking temperature and two RR charges of this black hole are

$$
\begin{aligned}
E & \sim \frac{L_{1} L_{2}}{g^{2} \alpha^{\prime 4}} r_{0}^{5}\left(\cosh 2 \alpha+\frac{7}{5}\right) \\
\Delta E_{b h} & \sim \frac{L_{1} L_{2}}{g^{2} \alpha^{\prime 4}} r_{0}^{5} \\
S_{b h} & \sim \frac{L_{1} L_{2}}{g^{2} \alpha^{\prime 4}} r_{0}^{6} \cosh \alpha \\
T & \sim \frac{1}{r_{0} \cosh \alpha} \\
Q_{1} & \sim \frac{L_{2}}{g \alpha^{\prime 3}} r_{0}^{5} \sinh 2 \alpha \cos \theta \\
Q_{2} & \sim \frac{L_{1}}{g \alpha^{\prime 3}} r_{0}^{5} \sinh 2 \alpha \sin \theta
\end{aligned}
$$

The matching point is

$$
r_{0} \sim \frac{\alpha^{\prime 1 / 2}}{(\cosh \alpha)^{1 / 2}}
$$

The spatial geometry outside the horizon is the "bottle neck" and is the same as that of the black 2-brane with D 2-brane charge, so there is no large gravitational field dressing. The system can be described by a single D-string winding around the $T^{2}$ diagonally with an angle $\theta$ with the $y_{1}$ direction and massless open strings ending on it. The winding number along the $y_{1}$ and $y_{2}$ directions are $Q_{1}$ and $Q_{2}$ respectively. The total length of the D-string is

$$
L_{t o t}=\sqrt{\left(Q_{1} L_{1}\right)^{2}+\left(Q_{2} L_{2}\right)^{2}} \sim \frac{L_{1} L_{2}}{g \alpha^{\prime 1 / 2}(\cosh \alpha)^{1 / 2}}
$$

Since $Q_{1}, Q_{2}$ are large numbers, we can think of the D-string as wrapped around the $T^{2}$ uniformly. The separation $d$ between adjacent turns is

$$
d=\frac{L_{1} L_{2}}{L_{t o t}} \sim g \alpha^{1 / 2}(\cosh \alpha)^{1 / 2}
$$

At temperature $T$, an open string that starts from the $i$-th turn of the D-string can end on any other $j$-th turn within $\alpha^{\prime} T$ distance. Since there is only one D-string, the different species of open stings are distinguished by $|i-j|$. So, the number of species is 
$\frac{\alpha^{\prime} T}{d}$. The entropy $S_{\text {open }}$ and the energy $E_{\text {open }}$ of this gas of open strings are

$$
\begin{aligned}
& S_{\text {open }} \sim \frac{\alpha^{\prime} T}{d} T L_{t o t} \sim \frac{L_{1} L_{2}}{g^{2} \alpha^{\prime} \cosh ^{2} \alpha} \sim S_{b h} \\
& E_{\text {open }} \sim T S_{\text {open }} \sim \frac{L_{1} L_{2}}{g^{2} \alpha^{\prime 3 / 2}(\cosh \alpha)^{5 / 2}} \sim \Delta E_{b h}
\end{aligned}
$$

So, they agree with the black hole entropy and excess energy.

In fact, without doing any calculation one can see that the correspondence principle should work for this black hole. As we have remarked, this black hole and the black hole described by (5.5) are related by a rotation in the $T^{2}$. Locally in the $y_{1}-y_{2}$ plane, there is no difference between the two. The entropies per unit area calculated in the open string gas picture are the same and the areas of the two $T^{2}$ are the same, therefore the total entropies calculated in this way must be the same.

If we go back to the original black hole (5.1), the picture at the matching point is that the 0-branes are dissolved into the 2-branes and become magnetic flux on the 2-branes, they form a bound state [16].

These black holes can obviously be generalized. One can start with a near extremal black p-brane in type II that carries a certain kind of RR charge, smear this black pbrane out in several other dimensions, make arbitrary rotations in the internal torus and finally T-dualize some of the internal directions if one wants to. All black holes with two RR charges in the category (A) that we classified in the introduction are included in this kind of constructions. The entropy of such black holes can all be understood by the correspondence principle.

\section{Discussions}

In this paper we have considered the entropy of near extremal black holes with multiple charges in the context of the correspondence principle [4] of Horowitz and Polchinski. We

have considered all black holes with two RR charges in the category $(\mathrm{A})$ and $(\mathrm{B})$ that are classified in the introduction and black holes with three or four RR charges. We have also considered a black hole with two RR charges and NS 5-brane charge. We found that the black hole entropy can always be counted for by the massless open strings on the D-branes for all cases except one. The exception is a four dimensional black hole with four RR charges, in which case the open string gas picture does not work because it contributes too small an entropy. We turned to the idea of brane-antibrane excitations for this case and 
found they contribute the correct entropy. But the calculation depends on the assumption that the branes and antibranes make up the entropy with the other three kinds of branes independently. The precise counting of entropy has been done for some of the black holes considered in this paper. Our results from the correspondence principle are consistent with the precise counting for those cases.

We have seen that the correspondence principle can be used to relate states of a large class of black holes to states of strings and D-branes. This can be thought of as a statistical understanding of the Bekenstein-Hawking entropy for black holes that have size of the string scale. It does not mean that we have a statistical understanding of the entropy for black holes that are much larger than the string scale. We do not yet understand why the black hole entropy does not change when we change the string coupling constant while keep the horizon area fixed in Planck units, i.e., why the black hole entropy is proportional to the horizon area measured in Planck units.

\section{Acknowledgments}

We would like to thank G. Horowitz for helpful discussions and reading of manuscript.

We would also like to thank R. Emparan, J. Pierre and J. Polchinski for helpful discussions. This work was supported in part by NSF Grant PHY95-07065. 


\section{References}

[1] L. Susskind, hep-th/9309145.

[2] E. Halyo, A. Rajaraman and L. Susskind, hep-th/9605112.

[3] E. Halyo, B. Kol, A. Rajaraman and L. Susskind, hep-th/9609075.

[4] G. T. Horowitz and J. Polchinski, Phys. Rev. D55 (1997) 6189, hep-th/9612146.

[5] J. Polchinski, Phys. Rev. Lett. 75 (1995) 4724, hep-th/9510017.

[6] H. Sheinblatt, hep-th/9705054.

[7] R. Emparan, hep-th/9704204; S. Das, hep-th/9705165; S. Mathur, hep-th/9706151; S. Das, S. Mathur, S. Rama and P. Ramadevi, hep-th/9711003; G. T. Horowitz and J. Polchinski, hep-th/9707170.

[8] A. Tseytlin, Nucl. Phys. B475 (1996) 149, hep-th/9604035; I. Klebanov and A. Tseytlin, Nucl. Phys. B475 (1996) 179, hep-th/9604166; M. Cvetic and A. Tseytlin, Nucl. Phys.B487(1996) 181, hep-th/9606033

[9] C. G. Callan, Jr. and J. M. Maldacena, Nucl. Phys. B472 (1996) 591, hep-th/9602043;

G. T. Horowitz and A. Strominger, Phys. Rev. Lett. 77 (1996) 2368, hep-th/9602051

[10] J. Maldacena and A. Strominger, Phys. Rev. Lett. 77 (1996) 428, hep-th/9603060.

[11] G. Horowitz, D. Lowe, and J. Maldacena, Phys. Rev. Lett. 77 (1996) 430, hepth/9603195.

[12] V. Balasubramanian and F. Larsen, Nulc. Phys. B478(1996) 199, hep-th/9604189.

[13] G. T. Horowitz, J. M. Maldacena, and A. Strominger, Phys. Lett. B383 (1996) 151, hep-th/9603109.

[14] N. Ohta and J. Zhou, hep-th/9706153

[15] J. Breckenridge, G. Michaud and R. Myers, Phys. Rev. D55 (1997) 6438, hepth/9611174.

[16] G. Lifschytz, Nucl. Phys. B499 (1997) 283, hep-th/9610125. 